\title{
A GENERALIZATION OF THE TARSKI-SEIDENBERG THEOREM, AND SOME NONDEFINABILITY RESULTS
}

\author{
LOU VAN DEN DRIES
}

This article points out some remarkable facts implicit in the results of Lojasiewicz [L1] and Gabrielov [Ga].

An important consequence of Tarski's work [T] on the elementary theory of the reals is a characterization of the sets which are elementarily definable from addition and multiplication on $\mathbf{R}$. Allowing arbitrary reals as constants, this characterization consists of the identification of the definable sets with the semialgebraic sets. (A semialgebraic subset of $\mathbf{R}^{m}$ is by definition a finite union of sets of the form $\left\{x \in \mathbf{R}^{m}: p(x)=0, q_{1}(x)>0, \ldots, q_{k}(x)>0\right\}$ where $p, q_{1}, \ldots, q_{k}$ are real polynomials.) The fact that the system of semialgebraic sets is closed under definability is also known as the Tarski-Seidenberg theorem, and this property, together with the topological finiteness phenomena that go with it - triangulability of semialgebraic sets [L2, Gi], generic triviality of semialgebraic maps [Ha]-make the theory of semialgebraic sets a useful analytic-topological tool.

Below we extend the system of semialgebraic sets in such a way that the Tarski-Seidenberg property, i.e., closure under definability, and the topological finiteness phenomena are preserved. The polynomial growth property of semialgebraic functions is also preserved. This extended system contains the arctangent function on $\mathbf{R}$, the sine function on any bounded interval, the exponential function $e^{x}$ on any bounded interval, but not the exponential function on all of $\mathbf{R}$. (And it couldn't possibly contain the sine function on all of $\mathbf{R}$ without sacrificing the finiteness phenomena, and a lot more.)

As a corollary we obtain that neither the exponential function on $\mathbf{R}$, nor the set of integers, is definable from addition, multiplication, and the restrictions of the sine and exponential functions to bounded intervals.

Questions of this type have puzzled logicians for a long time. (There still remain, of course, countless unsolved problems of this sort.) In a more positive spirit Tarski [T, p. 45] asked to extend his results so as to include, besides the algebraic operations on $\mathbf{R}$, certain transcendental elementary functions like $e^{x}$; the theorem below is a partial answer. (More recently, Hovanskii [Ho, p. 562] and the author [VdD1, VdD2] asked similar questions, and in [VdD3] we

Received by the editors May 7, 1986.

1980 Mathematics Subject Classification (1985 Revision). Primary $03 E 47$. 
present a program that, however, goes in a direction quite different from that of the present article.)

A precise formulation of our result requires the following notions.

(i) A set $X \subset \mathbf{R}^{m}$ is called semianalytic at the point $x \in \mathbf{R}^{m}$ if $x$ has an open neighborhood $U$ such that $U \cap X$ is a finite union of sets of the form

$$
\left\{y \in U: f(y)=0, g_{1}(y)>0, \ldots, g_{k}(y)>0\right\}
$$

where $f, g_{1}, \ldots, g_{k}$ are (real) analytic functions on $U$.

(ii) A set $X \subset \mathbf{R}^{m}$ is called semianalytic (in $\mathbf{R}^{m}$ ) if $X$ is semianalytic at each point $x$ in $\mathbf{R}^{m}$.

(iii) A set $X \subset \mathbf{R}^{m}$ is called subanalytic at the point $x \in \mathbf{R}^{m}$ if there is an open neighborhood $U$ of $x$ and a bounded semianalytic set $S \subset \mathbf{R}^{m+n}$, for some $n$, such that $U \cap X=\pi(S)$, where $\pi: \mathbf{R}^{m+n} \rightarrow \mathbf{R}^{m}$ is the obvious projection map.

(iv) A set $X \subset \mathbf{R}^{m}$ is called subanalytic (in $\mathbf{R}^{m}$ ) if $X$ is subanalytic at each point $x$ in $\mathbf{R}^{m}$.

(In (ii) and (iv) it is not enough to consider only points $x$ in $X$, but it suffices to consider points in the closure of $X$.)

It is clear that the semianalytic sets in $\mathbf{R}^{m}$ form a boolean algebra contained in the class of subanalytic sets in $\mathbf{R}^{m}$, and it is also easy to see that the latter class is closed under taking finite unions and finite intersections. Other facts relevant to us are:

(1) The subanalytic subsets of $\mathbf{R}$ and $\mathbf{R}^{2}$ are exactly the semianalytic subsets of $\mathbf{R}$ and $\mathbf{R}^{2}$. (For $m>2$ there are subanalytic sets in $\mathbf{R}^{m}$ which are not semianalytic.)

(2) A bounded semianalytic set has only finitely many connected components, and each component is also semianalytic.

(3) The bounded subanalytic sets in $\mathbf{R}^{m}$ are exactly the sets of the form $\pi(S)$ where $S$ is a bounded semianalytic set in $\mathbf{R}^{m+n}$, and $\pi: \mathbf{R}^{m+n} \rightarrow \mathbf{R}^{m}$ is the obvious projection map. Hence, by (2), a bounded subanalytic set has only finitely many connected components, and each component is also subanalytic. Also, if $X \subset \mathbf{R}^{m+1}$ is bounded and subanalytic, then its projection in $\mathbf{R}^{m}$ is subanalytic.

(4) The complement in $\mathbf{R}^{m}$ of a subanalytic set in $\mathbf{R}^{m}$ is subanalytic.

The theory of semianalytic sets, and (1) and (2), are due to Lojasiewicz [L1]; (3) is an exercise, and the difficult theorem (4) is due to Gabrielov [Ga].

The systems of semianalytic sets and of subanalytic sets share many good properties with the system of semialgebraic sets, but are not closed under definability. Surprisingly, we can give a small twist to the situation and recover closure under definability. Namely, call a set $X \subset \mathbf{R}^{m}$ finitely subanalytic if its image under the (semialgebraic) map from $\mathbf{R}^{m}$ to $\mathbf{R}^{m}$ :

$$
\left(x_{1}, \ldots, x_{m}\right) \mapsto\left(x_{1} / \sqrt{1+x_{1}^{2}}, \ldots, x_{m} / \sqrt{1+x_{m}^{2}}\right)
$$

is subanalytic in $\mathbf{R}^{m}$. (Note that this map is an analytic isomorphism onto the bounded open set $(-1,1)^{m}$, so that the finitely subanalytic sets are subanalytic.)

Our basic result - almost obvious, once observed - is the following. 
THEOREM. Let $F S_{m}$ be the class of finitely subanalytic sets in $\mathbf{R}^{m}$. Then $\left(F S_{m}\right)_{m \in \mathbf{N}}$ is an $O$-minimal Tarski system on $\mathbf{R}$.

A Tarski system (on $\mathbf{R}$ ) is a sequence $\left(\mathscr{S}_{m}\right)_{m \in \mathbf{N}}$ such that for each $m \in \mathbf{N}$ :

(T1) $\mathscr{S}_{m}$ is a boolean algebra of subsets of $\mathbf{R}^{m}$,

(T2) $X \in \mathscr{S}_{m} \Rightarrow \mathbf{R} \times X$ and $X \times \mathbf{R}$ are in $\mathscr{S}_{m+1}$,

(T3) $\left\{\left(x_{1}, \ldots, x_{m}\right): x_{1}=x_{m}\right\} \in \mathscr{S}_{m}$,

(T4) $X \in \mathscr{S}_{m+1} \Rightarrow \pi(X) \in \mathscr{S}_{m}$, where $\pi: \mathbf{R}^{m+1} \rightarrow \mathbf{R}^{m}$ is the projection on the first $m$ coordinates.

A Tarski system $\left(\mathscr{S}_{m}\right)$ on $\mathbf{R}$ is called a $O$-minimal if for each $r \in \mathbf{R}$ the singleton $\{r\}$ belongs to $\mathscr{S}_{1}$, the set $\left\{(x, y) \in \mathbf{R}^{2}: x<y\right\}$ belongs to $\mathscr{S}_{2}$, and the only sets in $\mathscr{S}_{1}$ are the subsets of $\mathbf{R}$ with only finitely many connected components - in other words, the finite unions of intervals and points.

The theorem is immediate from the definitions and properties (3) and (4) of subanalytic sets.

The theorem should be considered in combination with general facts about $O$-minimal Tarski systems which we explain now. First, a Tarski system $\left(\mathscr{S}_{m}\right)$ is closed under definability. Modulo familiar arguments this amounts to showing that if $X \in \mathscr{S}_{m}$ and $i(1), \ldots, i(m)$ are any positive integers $\leqslant n$, then the set

$$
Y=\left\{\left(y_{1}, \ldots, y_{n}\right) \in \mathbf{R}^{n}:\left(y_{i(1)}, \ldots, y_{i(m)}\right) \in X\right\}
$$

belongs to $\mathscr{S}_{n}$. Let us do this for the special case $X \in \mathscr{S}_{2}$ and $Y=X^{-1}=$ $\left\{\left(y_{1}, y_{2}\right):\left(y_{2}, y_{1}\right) \in X\right\}$. Just note that $\left(y_{1}, y_{2}\right) \in Y \Leftrightarrow \exists y_{3} \exists y_{4}\left(\left(y_{3}, y_{4}\right) \in X\right.$ $\left.\wedge y_{3}=y_{2} \wedge y_{4}=y_{1}\right)$. The part to the right of $\exists y_{4}$ defines a set in $\mathscr{S}_{4}$ : for instance, the relation $\left(y_{3}, y_{4}\right) \in X$ defines the set $\mathbf{R}^{2} \times X$, which is in $\mathscr{S}_{4}$ by (T2). The quantifier $\exists y_{4}$ amounts to projecting from $\mathbf{R}^{4}$ to $\mathbf{R}^{3}$, and $\exists y_{3}$ projects the resulting set into $\mathbf{R}^{2}$. Hence $Y \in \mathscr{S}_{2}$ by two applications of (T4). (The Kuratowski-Tarski translation of logical formulas into intersection, complement, projection operations, etc., is of course familiar to logicians, but this routine sort of "linguistic" argument seems little known to mathematicians in general. Perhaps this explains why one sometimes finds explicit proofs that the closure of a semialgebraic set is semialgebraic (and similar things) instead of a brief remark that this is immediate from the $\varepsilon-\delta$ formulation of closedness.)

Some nontrivial facts about arbitrary $O$-minimal Tarski systems were obtained in [VdD2, P-S, K-P-S, VdD4], but, except for the already familiar systems of piecewise linear sets, and of semialgebraic sets, no other interesting $O$-minimal Tarski systems were known at the time: it seems that the system of finitely subanalytic sets is the first known $O$-minimal Tarski system on $\mathbf{R}$ that properly extends the system of semialgebraic sets. (It is not known whether there is any Tarski system strictly between the system of piecewise linear sets and the system of semialgebraic sets.)

Here are some " $O$-minimal" finiteness results.

Let $\mathscr{S}=\left(\mathscr{S}_{m}\right)$ be an $O$-minimal Tarski system on $\mathbf{R}$. We say that a map $f$ : $X \rightarrow Y, X \subset \mathbf{R}^{m}, Y \subset \mathbf{R}^{n}$, belongs to $\mathscr{S}$ if its graph is in $\mathscr{S}_{m+n}$.

PIECEWISE MONOTONICITY; cf. [VdD2, §2]. If $(a, b)$ is an interval and $f$ : $(a, b) \rightarrow \mathbf{R}$ belongs to $\mathscr{S}$ then there are $a_{0}<a_{1}<\cdots<a_{m}, a_{0}=a, a_{m}=b$, 
such that on each subinterval $\left(a_{i}, a_{i+1}\right)$ the function is either continuous and strictly monotone, or constant.

UNIFORM BOUNDS; cf. [K-P-S]. Each set $Y \in \mathscr{S}_{m}$ has only finitely many connected components, and each component also belongs to $\mathscr{S}_{m}$. If further $f$ : $Y \rightarrow \mathbf{R}^{n}$ belongs to $\mathscr{S}_{m+n}$, then there is a positive integer $N=N(f)$ such that for each $x \in \mathbf{R}^{n}$, the fiber $f^{-1}(x)$ has at most $N$ components.

Triangulability; cf. [VdD4], generalizing the proof in [Hi]. Let $\mathscr{S}$ contain (the graphs of) addition and multiplication, and let $X_{1}, \ldots, X_{k} \in \mathscr{S}_{m}$. Then there is a finite simplicial complex $K$ in $\mathbf{R}^{m}$ and a homeomorphism $h$ in $\mathscr{S}$ between $X_{1} \cup \cdots \cup X_{k}$ and a subset of $|K|$ such that each $h\left(X_{i}\right)$ is a union of (open) simplices of $K$.

GENERIC TRIVIALITY; cf. [Ha] for the semialgebraic case. Let $\mathscr{S}$ contain addition and multiplication, and let $f: X \rightarrow Y$ be a map in $\mathscr{S}, Y \neq \varnothing$. Then $Y$ has a subset $Z$ in $\mathscr{S}$ of lower dimension such that over each of the finitely many connected components $C$ of $Y \backslash Z$ the map $f$ is $\mathscr{S}$-trivial, that is, taking $c \in C$ there is a homeomorphism $f^{-1}(C) \simeq C \times f^{-1}(c)$ in $\mathscr{S}$, that commutes with the obvious maps onto $C$.

From a model-theoretic viewpoint generic triviality follows easily from triangulability; cf. [VdD4].

A few remarks on what is finitely subanalytic and what is not. Clearly, all bounded subanalytic sets (and hence their complements) are finitely subanalytic, and in fact, the system of finitely subanalytic sets is the Tarski system generated by addition, multiplication, and the bounded semianalytic sets. More precisely, the theory of the ordered field $\mathbf{R}$ with predicates for all bounded semianalytic sets is model complete, and the sets definable in this structure are exactly the finitely subanalytic sets. Every restriction of an analytic function $f: U \rightarrow \mathbf{R}, U$ open in $\mathbf{R}^{m}$, to a compact subanalytic set $X \subset U$, is finitely subanalytic. (That is, the graph of $f \mid X$ is finitely subanalytic.) The arctangent on $\mathbf{R}$ is finitely subanalytic by its definition as the inverse of the tangent on $(-\pi / 2, \pi / 2)$, and on this interval the tangent is definable from sine and cosine.

PolynOMIAL GROWTH. Given a finitely subanalytic function $f:(0, \infty) \rightarrow \mathbf{R}$, there is $d \in \mathbf{N}$ and $a>0$ such that $|f(t)|<t^{d}$ for $t>a$.

To see this, use the transformation $t=1 / x$ in the domain, and the same transformation or a translation in the codomain to obtain a function $g$ : $(0, \varepsilon) \rightarrow \mathbf{R}$ with subanalytic graph and $g(x) \rightarrow 0$ as $x \rightarrow 0$. By (1), the graph of $g$ is even semianalytic at $(0,0)$, hence there is a nonzero real power series $F(X, Y)=\sum a_{m n} X^{m} Y^{n}$ converging in a neighborhood of $(0,0)$ such that $F(x, g(x))=0$ for all small positive $x$. Assuming $g$ is not identically zero near 0 we may divide $F$ by a suitable power of $X$ and obtain a new $F$ that is not divisible by $X$. Hence, by Weierstrass preparation, we may even assume:

$$
F=Y^{k}+a_{1}(X) Y^{k-1}+\cdots+a_{k}(X),
$$

where the $a_{i}(X)$ are real power series with $a_{i}(0)=0$ and converging near zero. Then $F(x, g(x))=0$ and continuity of $g$ near 0 gives us a Puiseux expansion (with real coefficients) $c_{1} x^{1 / k}+c_{2} x^{2 / k}+\cdots$ converging to $g(x)$ for small positive $x$. Translated back to $f$ this means that, asymptotically, $f(t) \sim c \cdot t^{r}$ as $t \rightarrow \infty$, for a real constant $c$ and a rational constant $r$. 
Note. This proof shows also that for irrational $r \in \mathbf{R}$ the function $x^{r}$ on $(0, \infty)$ is not finitely subanalytic.

\section{REFERENCES}

[Ga] A. Gabrielov, Projections of semi-analytic sets, Functional Anal. Appl. 2 (1968), 282-291. (Russian)

[Gi] B. Gieseke, Simpliziale Zerlegung abzählbarer analytische Räume, Math. Z. 83 (1964), 177-213.

[Ha] R. Hardt, Semi-algebraic local triviality in semi-algebraic mappings, Amer. J. Math. 102 (1980), 291-302.

[Hi] H. Hironaka, Triangulation of algebraic sets, Algebraic Geometry, Arcata 1974, Proc. Sympos. Pure Math., vol. 29, Amer. Math. Soc., Providence, R. I., 1975, pp. 165-186.

[Ho] A. Hovanskii, Fewnomials and Pfaff manifolds, Proc. ICM, Warsaw, 1983, pp. 549-564.

[K-P-S] J. Knight, A. Pillay, and C. Steinhorn, Definable sets in ordered structures. II, Trans. Amer. Math. Soc. 295 (1986), 593-605.

[L1] S. Lojasiewicz, Ensembles semi-analytiques, mimeographed notes, Inst. Hautes Études Sci., 1965

[L2] 449-474. Triangulation of semi-analytic sets, Ann. Sci. École Norm. Sup. Pisa (3) 18 (1964),

[P-S] A. Pillay and C. Steinhorn, Definable sets in ordered structures. I, Trans. Amer. Math. Soc. 295 (1986), 565-592.

[T] A. Tarski, A decision method for elementary algebra and geometry, (2nd ed. revised), Rand Corporation monograph, Berkeley and Los Angeles, 1951.

[VdD1] L. van den Dries, Analytic Hardy fields and exponential curves in the real plane, Amer. J. Math. 106 (1984), 149-167.

[VdD2] _ Remarks on Tarski's problem concerning ( $\mathbf{R},+, \cdot$, exp), Logic Colloquium, 1982, pp. 97-121, (G. Lolli, G. Longo and A. Marcja, eds.), North-Holland, 1984.

[VdD3] _ Tarski's problem and Pfaffian functions, Proc. Logic Colloquium, 1984 (to appear).

[VdD4] lectures at University of Konstanz.

Max-Planck-Institut für Mathematik, Gottfried-Claren-Strasse 26, 5300 Bonn 3, FEDERAL RePUblic OF Germany

Current address: Department of Mathematics, University of Illinois at Urbana-Champaign, Urbana, Illinois 61801 
